\section{Scintigraphy-based analysis of possible pulmonary lesions after foam sclerotherapy: a pilot study}

\author{
Lorenzo Tessari, ${ }^{1}$ Marcello Izzo, ${ }^{2}$ \\ Attilio Cavezzi, ${ }^{3}$ Francesco Zini, ${ }^{4}$ \\ Mirko Tessari, ${ }^{5}$ Daniela Grigolato ${ }^{6}$ \\ ${ }^{1}$ Glauco Bassi Foundation, Trieste; \\ 2Mathematics for Technology, Medicine \& \\ Biosciences Research Center, University \\ of Ferrara; ${ }^{3}$ Eurocenter Venalinfa, San \\ Benedetto del Tronto (AP); ${ }^{4}$ Casa di Cura \\ Città di Parma, Parma; ${ }^{5}$ Vascular Disease \\ Center, University of Ferrara; \\ 'UOC of Nuclear Medicine, University \\ Hospital of Verona, Italy
}

\section{Abstract}

The aims of this study were to assess extempo-

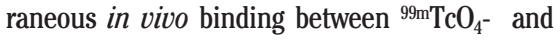
two sclerosant detergents in foam sclerotherapy, and subsequently to control any possible damage in lungs and other organs related to sclerosant foam passage. A prospective comparative pilot study was performed on two male patients (62 and 56 years old) affected by varicose veins; each of them underwent scintigraphy investigations with free radiotracer and a scintigraphy investigation after each of the four sessions of sclerotherapy of varicose tributaries of the lower limbs with labeled sclerosant foam. One of the two patients underwent two further scintigraphic investigations, with free radiotracer and with labeled sclerosant foam, at a later stage. Four mL of $2 \%$ polidocanol (POL) foam, or four $\mathrm{mL}$ of $1 \%$ sodiumtetradecylsulfate (STS) foam for session were injected. The sclerosant foam was labeled with the radioactive tracer technetium pertechnetate, ${ }^{99} \mathrm{TcO}_{4^{-}}(120 \mathrm{MBq}$ per exam). Two scintigraphy assessments for free tracer (basal) and five scintigraphy investigations of bound-tosclerosant tracer uptake/transit were obtained. No relevant variations in time/activity curves of the lungs and other organs were documented between the basal and post-sclerotherapy findings, also at the later stage. Free radiotracer mean region-of-interest data were: 336 counts (heart), 208 counts (lungs) and 371 counts (thyroid). Mean values extrapolated from each curve at each step for labeled $\mathrm{CO}_{2} \mathrm{O}_{2}$-based sclerosant foam were respectively: 351 counts (POL) and 328 counts (STS) for heart, 202 counts (POL) and 188 counts (STS) for lungs, 335 (POL) and 263 (STS) for thyroid. No pulmonary damage by sclerosant foam was caused. Neither immediately after treatments, nor at short-term follow-up.

\section{Introduction}

Foam sclerotherapy was introduced more than 60 years ago, and Cabrera and Monfreux's innovative methods in the late 1990s gave a new impulse to using sclerosant foam (SF). ${ }^{1}$ The extended use of this treatment was achieved after the introduction of the Tessari method. ${ }^{2,3}$

Foam sclerotherapy has proved to be a validated, effective and safe method to treat varicose veins of the lower limbs, ${ }^{4}$ and possibilities and limitations for this technique have been elucidated in several publications..$^{5,6}$ More recently, issues have been raised and discussed as to potential risks, side effects and complications of SF. Distal embolization of SF bubbles to lungs and possible lung capillary/parenchyma inflammation has been postulated in the past ${ }^{8}$ and recent ${ }^{9}$ literature. Furthermore, especially in the presence of a right-to left shunt such as a patent foramen ovale, isolated cases of neurological complications have been reported. ${ }^{4}$

The authors aimed to assess any possible change in the tissues of various organs, particularly the lungs, which may be affected by possible passage/impact of SF bubbles. Furthermore, any difference between airbased $\mathrm{SF}$ and $\mathrm{CO}_{2} \mathrm{O}_{2}$-based SF was investigated in order to assess a possible higher safety profile of biocompatible gases vs room air. Finally, the clinical and experimental trial aimed at assessing any difference between the two commonly used detergent sclerosant drugs [sodiumtetradecysulfate (STS) and polidocanol (POL)] as to the above-mentioned issues. In order to get a reproducible and quantifiable method for detecting possible tissue changes in the target/transit organs after sclerosant foam injections, scintigraphy was employed in a qualitative and semi-quantitative way. On the basis of the chemical/physical properties of pertechnetate ion $\left({ }^{99} \mathrm{TcO}_{4}-\right)$, a protocol to assess extemporaneous in vivo binding between ${ }^{99} \mathrm{TcO}_{4^{-}}$and POL/STS foam during various steps was designed. $\mathrm{P}$

${ }^{99} \mathrm{TcO}_{4^{-}}$shows a stable chemical structure not easily suited to bind other molecules. The study aimed at assessing the possibility of linkage between this radiotracer and the SF to check the presumed possibility of tracing the pathway and accumulation of SF bubbles by means of ${ }^{99} \mathrm{TcO}_{4^{-}}$, as reported in a previous publication. ${ }^{8}$ In fact, in the vascular stream Pertechnetate ions remain in equilibrium, partially in free form and partially bound to serum proteins. More specifically, free ions leave the vascular compartment and migrate to interstitial fluids as a result of their small size, so as to decrease the pertechnetate blood concentration This leads to an equiva-
Correspondence: Lorenzo Tessari, via Giovanni Falcone 24/B, Peschiera del Garda (VR), 37019 Italy.

Tel.: +39.0456401681 .

E-mail: lorenzo@tessaristudi.it

Key words: foam sclerotherapy, scintigraphy.

Contributions: LT, study design, data collection, data analysis, critical review, final approval; MI, data analysis, critical review, final approval; AC, data collection, data analysis, writing, critical review, final approval; FZ, data collection, data analysis, final approval; MT, data collection, final approval; DG, data analysis, critical review, writing, final approval.

Conflict of interests: the authors declare no potential conflict of interests.

Received for publication: 24 April 2014.

Revision received: 23 September 2014.

Accepted for publication: 24 September 2014.

This work is licensed under a Creative Commons Attribution 3.0 License (by-nc 3.0).

(C) Copyright L. Tessari et al., 2014

Licensee PAGEPress, Italy

Veins and Lymphatics 2014; 3:4037

doi:10.4081/vl.2014.4037

lent release of ${ }^{99} \mathrm{TcO}_{4}$ - that is bound to the proteins as an unavoidable result of its elimination. ${ }^{10}$ The Pertechnetate ion is removed by various organs and systems into the interstitial liquid, as seen in the stomach, thyroid, salivary glands, bowel, choroid plexus, kidneys, vascular structures and mucosae. ${ }^{11}$ Interestingly, the pertechnetate ion is strongly taken up by the thyroid gland only in its free form and not bound to other molecules. ${ }^{12}$

Noticeably, lungs represent a transit organ for ${ }^{99 \mathrm{~m}} \mathrm{TcO}_{4^{-}}$and its temporal concentration peak declines along the $30 \mathrm{~min}$ of the scintigraphic observation and this pattern is very similar also for other various organs. ${ }^{13}$ Finally and more importantly, the increased capillary permeability leads to an increased ${ }^{99} \mathrm{mcO}_{4}$ uptake in inflamed tissues. Therefore, in the case of possible drug-induced lung damage following foam sclerotherapy, there would be an increased ${ }^{99} \mathrm{TcO}_{4}$ - persistence into the lungs after its injection during the post-treatment period.

\section{Materials and Methods}

A prospective comparative pilot study was performed on two male patients (56 and 62 years old) affected by primary varicose veins (C2 of CEAP - Clinical-Etiology-AnatomicPathophysiologic - classification) related to 
great saphenous vein (GSV) reflux. The patients were treated with SF which was labeled with a radionuclide $\left({ }^{99 \mathrm{~m}} \mathrm{TcO}_{4}-\right)$ and they gave informed written consent to the procedures; no ethical committee approval was required, as per any observational study in Italy. Basal scintigraphy was performed immediately after injection of $4 \mathrm{~mL}$ of saline solution with the radiotracer (RT) ${ }^{99} \mathrm{TcO}_{4}$ - in a varicose tributary of the lower leg. Subsequently, scintigraphy was performed after each of the four sessions of foam sclerotherapy which were performed on each patient. An overall imaging of the outcomes is reported in Figure 1. Injections were performed into the tributaries and the GSV stems, tailoring each session according to the outcomes achieved, using $4 \mathrm{~mL}$ of $2 \%$ POL foam or $4 \mathrm{~mL}$ of $1 \%$ STS foam per session with the patient in the supine position. Sclerosant foam was obtained through Tessari's method ${ }^{2}$ with one part of sclerosant drug (POL or STS) and four parts of gas (air or $\mathrm{CO}_{2} 70 \%+\mathrm{O}_{2} 30 \%$ ). Prior to foam formation, the sclerosant drug was mixed with ${ }^{99} \mathrm{TcO}_{4^{-}}(120 \mathrm{MBq}$ per exam): more specifically $1 \mathrm{cc}$ of POL $2 \%$, or $1 \mathrm{cc}$ of STS $1 \%$ were mixed with 0.2 cc of RT.

In more detail, the flow chart of the protocol for each patient was as follows:

- Step 1, basal scintigraphy examination

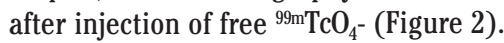

- Step 2 at day 7, scintigraphy examination 10 min after POL $2 \%$ + air radiolabelled sclerosant foam injection (Figure 3A).

- Step 3 at day 14 , scintigraphy examination 10 min after POL $2 \%+\mathrm{CO}_{2} \mathrm{O}_{2}$ radiolabeled sclerosant foam injection (Figure 3B).

- Step 4 at day 21, scintigraphy examination 10 min after STS $1 \%+\mathrm{CO}_{2} \mathrm{O}_{2}$ radiolabeled sclerosant foam injection (Figure 3C).

- Step 5 at day 28, scintigraphy examination 10 min after STS $1 \%$ + air radiolabeled sclerosant foam injection (Figure 3D).

- Step 6 at day 42, scintigraphy examination for one of the two patients during the 90 min following free RT injection and $90 \mathrm{~min}$ after STS 1\% + air radiolabeled sclerosant foam injection (Figure 4). This final step was undertaken to assess any possible late lung (and thyroid) tissue change due to several/repeated foam sclerotherapy sessions, as in the previous steps.

Radiation exposition was controlled in each patient after every step and no limitexceeding figure was recorded.

Uptake and transit of free RT and of sclerosant-bound RT were assessed in transit organs (lungs and heart) and in target organs (thyroid, salivary glands, stomach) with semiquantitative scintigraphy by means of a double-head gamma camera and region-of-interest (ROI) investigation. All scans lasted 30 min except for the step 6 scan (90 min). All dynamic evaluations were focused on the chest and neck, in anterior and posterior projections.

Ninety photograms (1800 $\mathrm{s}$ in total) were taken at intervals of $20 \mathrm{~s}$ every frame (matrix $128 \times 128$ pixel). The count per second in ROIs were plotted against time and the curves were fitted (counting out the initial transient phase).

Activity in the site of sclerosant injection was measured at different interval times (see above) and the total count was divided by the background activity registered in the contralateral leg.

\section{Results}

At step 1 after injection of pertechnetate ion, the two patients showed a typical pattern of RT distribution in all transit and target organs and more specifically the semi-quantitative calculations resulted in a mean half time of $57.26 \mathrm{~s}$ in the lung, $57.35 \mathrm{~s}$ in the heart and $-138.68 \mathrm{~s}$ in the thyroid. The integrated areas between $9^{\text {th }}$ and $30^{\text {th }}$ min were 336 (heart), 208 (lung) and 371 (thyroid). For each patient at step 1 , step 2 , step 3 and step 4 , step 5 the various ROI images (same size) and relative

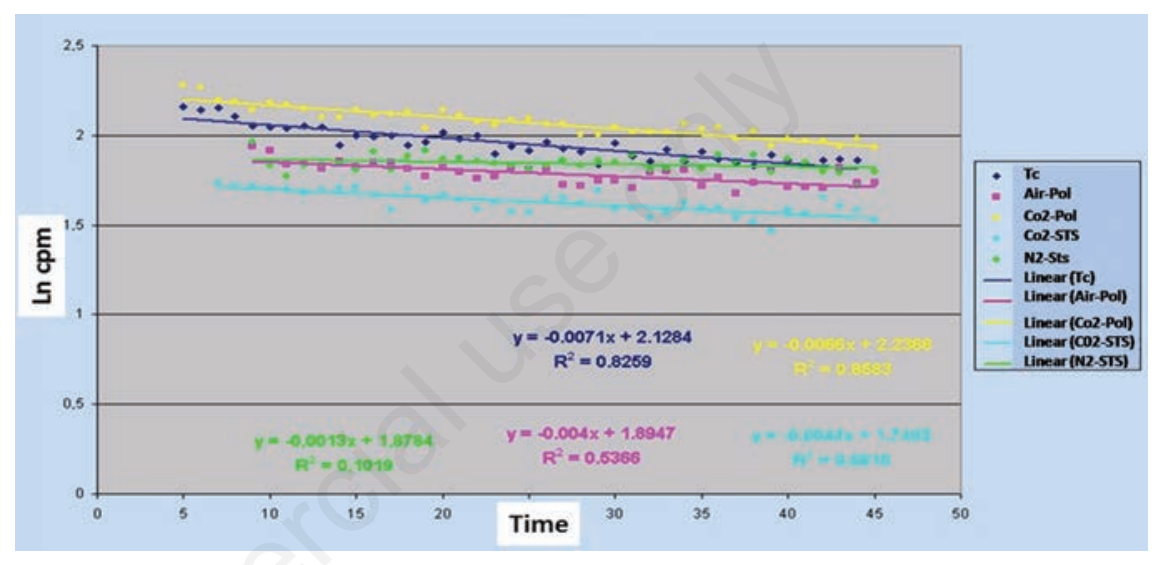

Figure 1. Curves and images of various regions-of-interest of different organ uptake afterfree radiotracer injection (step 1).

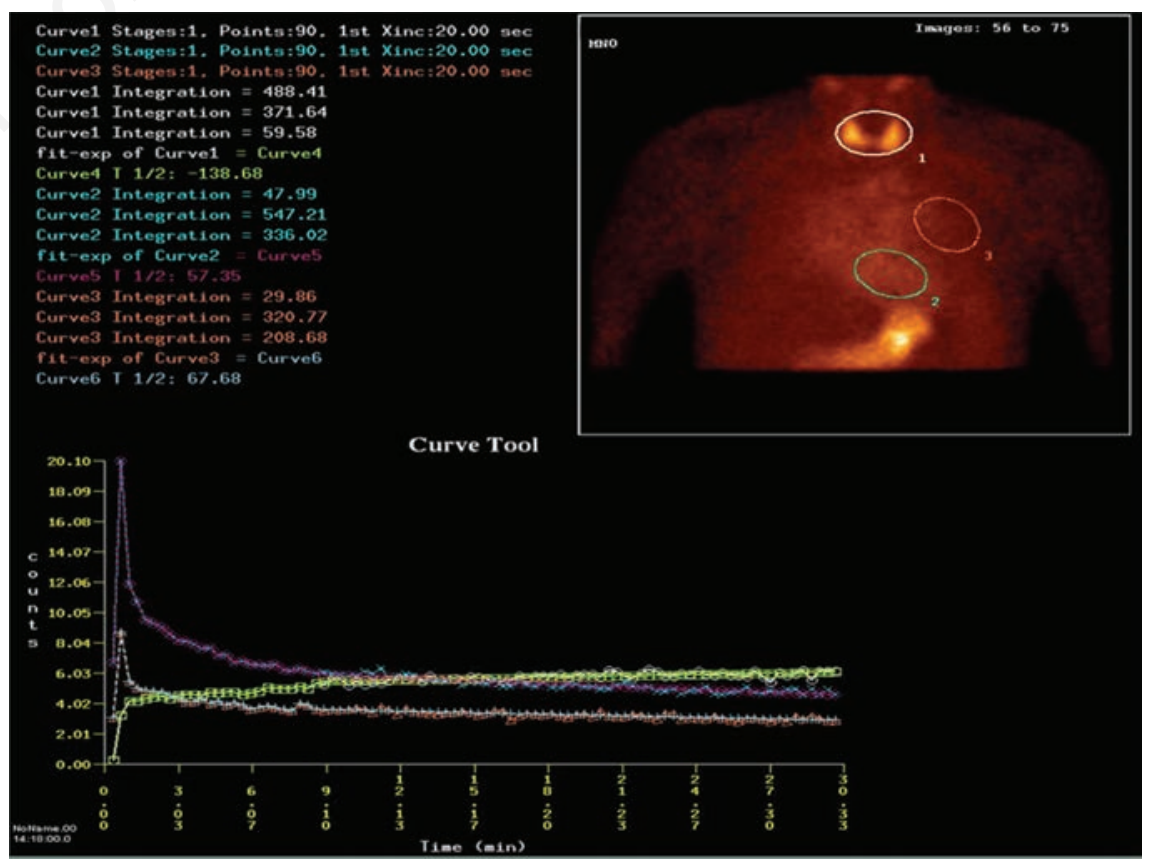

Figure 2. Organ uptake at step 1 for one patient. 
curves of uptake have been summarized in Table 1 where mean figures for each organ and each session are summarized. The mean figures have been calculated for each organ after summation of all resulting values for each step and from both patients.

Comparison between step 1 and steps 2, 3, 4 and 5 showed overlapping time/activity curves related to lung transit. Assessment of overall amplitude of the curves showed a minimal difference in comparison to free RT curve related to the radioactivity which has remained trapped in the vessels of the leg which was the site of the sclerosant foam injection.

The only relevant difference between the basal step 1 and all other foam sclerotherapy steps (2 to 5) concerned the delayed transit time and a small reduction in amplitude in all samples, which is a consequence of peripheral vein uptake of the RT mixed with sclerosant foam. In fact, spasm of the treated vein and sclerosant foam stagnation in the treated veins (varicose tributary and consequently GSV trunk) contributed to the delayed arrival of RT to the lungs. Imaging of the varicose tributary and saphenous veins (Figure 5A) clearly depicted RT stagnation in these peripheral vein territories and gradual ${ }^{99 \mathrm{~m} \mathrm{TcO}_{4^{-}}}$release over time (Figure 5).

The activity/time curves related to the main up taking organs (thyroid and stomach in step 2,3,4 and 5 showed a pattern distribution which was overlapping the free RT curve of step 1. Data on these dose uptakes are summarized in Figure 1 and lung/thyroid/heart values are reported in Table 1.

As thyroid normally takes up ${ }^{99} \mathrm{TcO}_{4}$ - in its free form, the findings above highlight how RT in thyroid circulation is neither bound to the sclerosant bubbles nor to the sclerosant drug.

Examination of any possible difference in the patterns and curves for the two sclerosant drugs STS and POL at the four different sclerotherapy steps (2 to 5) in comparison to step 1 (free RT) showed overall similar results.

Conversely, air-based SF showed a more prolonged delay in time overall to reach the maximum radioactivity in the curves (time to max) in comparison to $\mathrm{CO}_{2}+\mathrm{O}_{2}$-based $\mathrm{SF}$ (Table 2).
We can speculate that bubbles of $\mathrm{CO}_{2}+\mathrm{O}_{2}$ sclerosant foam tend to break up earlier than airbased SF so that RT release is accelerated.

Data analysis of the procedures of step 6 in one of the two investigated patients showed free ${ }^{99 \mathrm{~m}} \mathrm{TcO}_{4}$ - curves at the pulmonary and thyroid levels which overlap the step 1 activity/time curves (Figure 4). As no signifi-
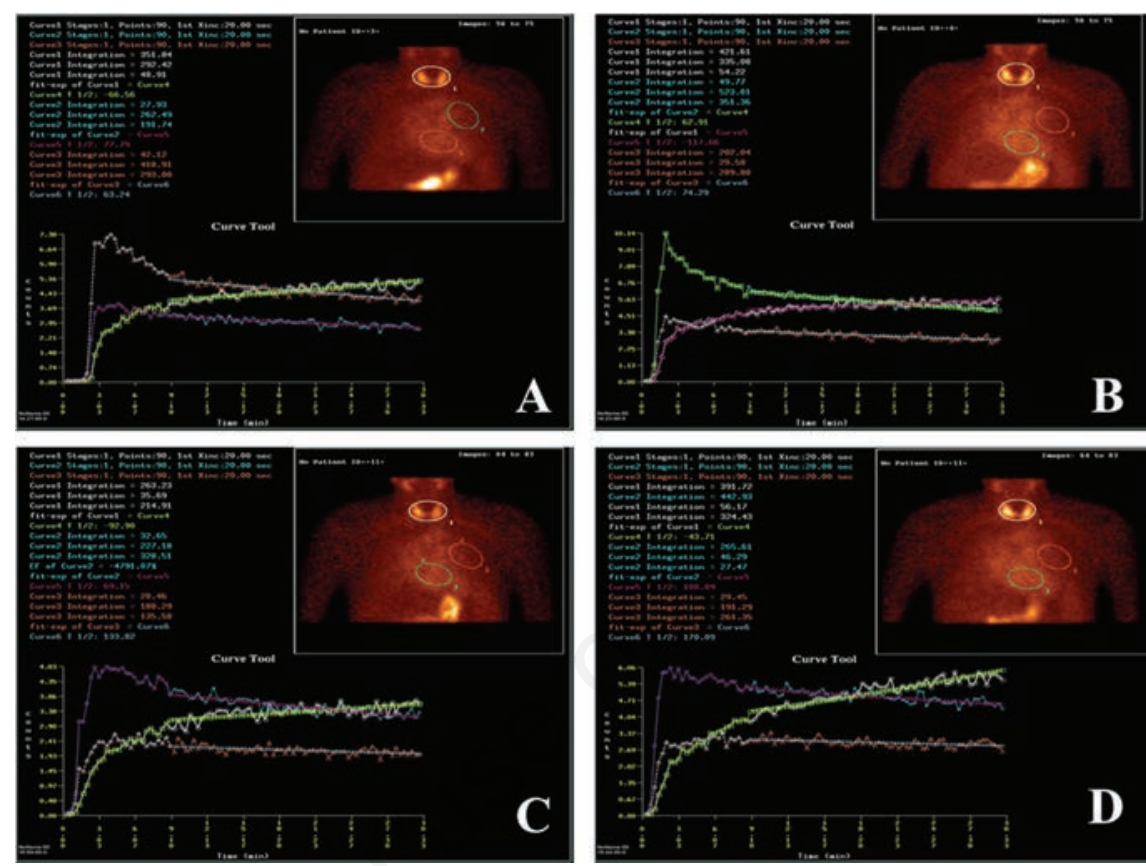

Figure 3. A) Organ uptake at step 2 for one patient; B) organ uptake at step 3 for one patient; C) organ uptake at step 4 for one patient; D) Organ uptake at step 5 for one patient.
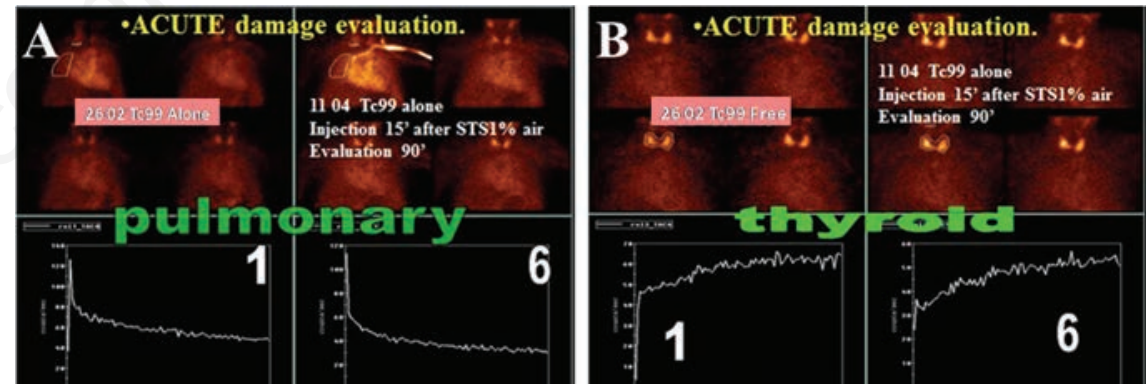

Figure 4. A) Step 6 images and curves of lungs after free radiotracer (RT) injection (left) and after last labeled sodiumtetradecylsulfate (STS) injection (right); B) step 6 images and curves of thyroid after free RT (left) and last labeled STS foam.

Table 1. Figures deriving from total counts in the different regions-of-interest between the $9^{\text {th }}$ and the $30^{\text {th }}$ min in each curve.

\begin{tabular}{|c|c|c|c|c|}
\hline & Heart & Lung & $\begin{array}{l}\text { Difference between } \\
\text { basal (step 1) and sclerosant } \\
\text { foam in lungs at step 2, 3, 4, } 5\end{array}$ & Thyroid \\
\hline${ }^{99} \mathrm{mcO}_{4}^{-}(\mathrm{BASAL})$ & 336.02 & 208.49 & - & 371.64 \\
\hline POL air & 293.00 & 191.74 & $-9 \%$ & 292.42 \\
\hline POL CO $\mathrm{O}_{2}$ & 351.36 & 202.04 & $-4 \%$ & 335.08 \\
\hline STS $\mathrm{CO}_{2} \mathrm{O}_{2}$ & 328.51 & 188.29 & $-10 \%$ & 263.23 \\
\hline STS air & 265.61 & 191.29 & $-9 \%$ & 324.43 \\
\hline
\end{tabular}

POL, polidocanol; STS, sodiumtetradecylsulfate. 
cant difference in uptake was highlighted, these data confirm that at 42 days after four sclerotherapy sessions, no relevant changes in lung and thyroid circulation/tissues occurred.

Furthermore, the second part of step 6 consisted in one last foam sclerotherapy session with STS 1\% sclerosant foam and subsequent scintigraphic assessment of RT uptake in the lungs and thyroid again showed no relevant changes in the curves in comparison to curves originating from free RT injection performed at the first part of step 6 (Figure 4).

As a consequence of these findings, no lung circulation changes due to sclerosant foam can be postulated.

The lack of important variations in the time/activity curves of the lungs between the first and the last foam sclerotherapy session indicates that sclerosant foam does not reach or does not induce any lung lesion.

\section{Discussion}

This observational experimental study was carried out to assess sclerosant foam effect on a few organs that may potentially receive a tissue lesion following sclerotherapy sessions.

After a preliminary investigation on technetium pertechnetate chemical-physical proprieties and on the possible binding interactions with SF, the authors finalized a clinical and scintigraphy protocol. The aim was to assess the pathway of SF bubbles/drug through the blood stream and the binding/effect of SF on target and transit organ tissues.

In a previous study, ${ }^{89 \mathrm{~m}} \mathrm{TcO}_{4}$ - was used to assess pulmonary patterns and bubble accumulation during foam sclerotherapy. In our study, the use of ${ }^{99} \mathrm{mcO}_{4}$ - proved to be unreliable to demonstrate the sclerosant drug pathway along the blood stream and within the transit organs (e.g. lungs), as the radiotracer flows independently. In fact, to bind the sclerosant drug to the ${ }^{99 \mathrm{~m}} \mathrm{Tc}$ we would need an adequate procedure of reduction ${ }^{99} \mathrm{TcO}_{4}-+\mathrm{Sn} 2++$ STS or POL and hence the extemporaneous way to combine sclerosant drug with RT proved to be of no utility.

From the chemical and scintigraphy point of view, it is recognized that radionuclide ${ }^{99 \mathrm{~m} T c}$ is obtained in physiologic solution as pertechnetate ion, and it has a coordination compound among technetium and oxygen. The metal atom is bound to four binding oxygen (0-2) thus forming a very compact tethraedron-like structure, with four identical faces of triangular shape.

Technetium oxidation state in ion is +7 . This constitutes the most stable oxidation state and represents one of the most stable pertechnetate chemical species in watery solutions.

In order to prepare a radiolabelled drug from the ${ }^{99 \mathrm{~m}} \mathrm{TcO}_{4}$, with coordinated bindings to give particular biological properties to the complex, it would be necessary to remove, partially or totally, the oxygen atoms that are bound to the metal. Finally substitution of these $\mathrm{O}_{2}$ atoms with the coordinated atoms of new binders cannot happen with a simple extemporaneous mixing. Hence tracing SF pathway by means of this radiolabelled ion would require a more complex process to overcome the chemical/physical difficulties as to above. Contrasting evidence from a few studies highlighted the possible alterations induced by the sclerosant foam on the lung capillaries/tissues. After a preliminary non-conclusive scintigraphic study from Milleret, ${ }^{8}$ on one side Grandi $^{9}$ and collaborators showed lung parenchyma lesions in rats after foam sclerotherapy, on the other side Moro ${ }^{14}$ and coworkers recently showed no significant changes in pulmonary ventilation parameters after sclerosant foam injection.

One of the objective limitation of Grandi's study ${ }^{9}$ subsides in the injected dose of SF in the rats which was by far much higher than the maximum permitted dose in humans; similarly the very short time/pathway between ears (the injected site) and lungs may have influenced their conclusions as well.

Notwithstanding the very small sample size, the variability of the methodology (gas,
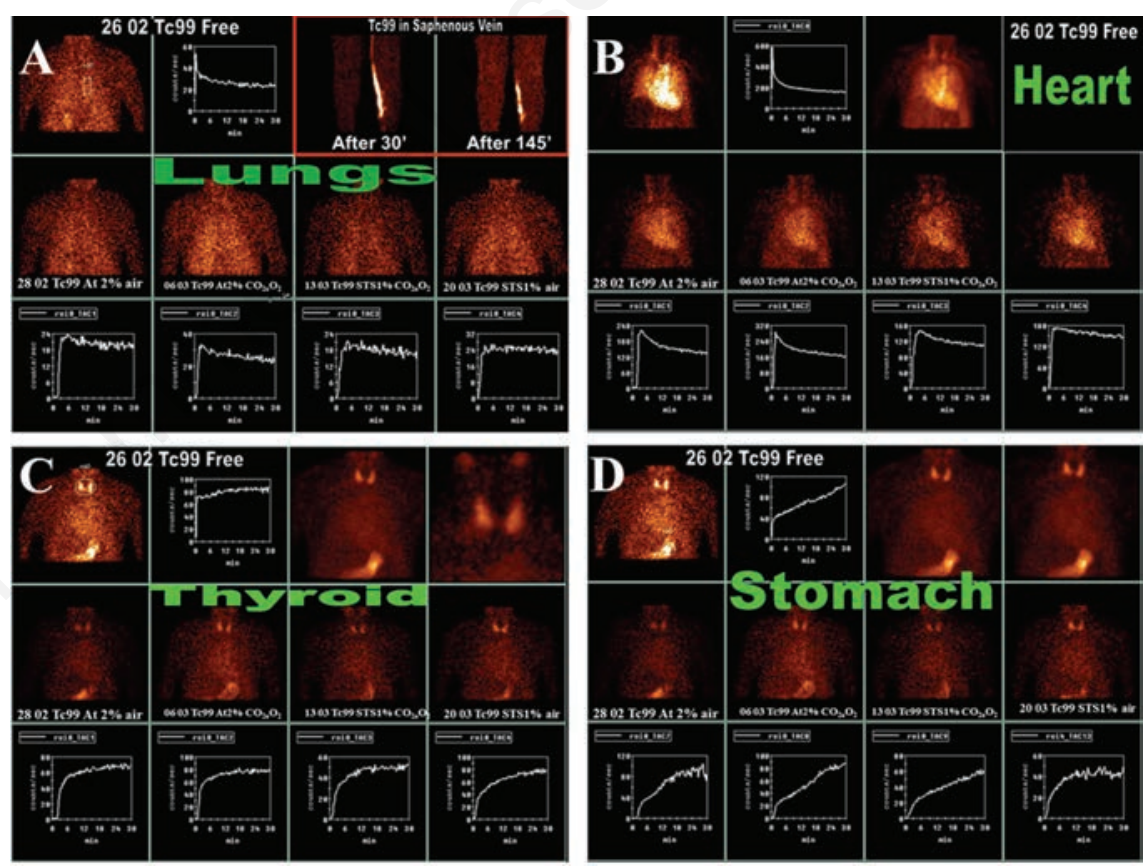

Figure 5. A) Lung and great saphenous vein imaging and lung uptake curves (for the first five steps); B) heart imaging and relative uptake curves (for the five steps); C) thyroid imaging and uptake curves (for the five steps); D) stomach imaging and uptake curves (for the five steps).

Table 2. Time to maximal radioactivity in each curve.

\begin{tabular}{lcccc} 
& Lungs & Heart & Thyroid & Background (Us contralateral limb) \\
$99 \mathrm{~m}^{2} \mathrm{TCO}_{4^{-}}$ & $60 \mathrm{~s}$ & $60 \mathrm{~s}$ & $27 \mathrm{~min}$ & $15 \mathrm{~min}$ \\
$\mathrm{CO}_{2} \mathrm{O}_{2}$ based SF & $120 \mathrm{~s}$ & $120 \mathrm{~s}$ & $17 \mathrm{~min}$ & $19 \mathrm{~min}$ \\
\hline Air-based SF & $5 \mathrm{~min}$ & $4 \mathrm{~min}$ & $26 \mathrm{~min}$ & $26 \mathrm{~min}$ \\
\hline
\end{tabular}

SF, sclerosant foam. 
agents) and the limited pathology treated, this preliminary study confirms the overall safety of foam sclerotherapy as to the possible chemical lesion to transit/target organs. The invariable and total neutralization of STS or POL drug by blood components (proteins firstly) was demonstrated by Parsi ${ }^{15-17}$ and Watkins. ${ }^{18}$ Similarly Tessari and collaborators showed no free STS in the blood of common femoral vein (at short/mid/long term time intervals) after injecting STS-based sclerosant foam in the great saphenous vein/tributaries of the lower leg. ${ }^{19}$ Due to the very small sample size no statistical analysis was performed, though all data seem to be coherent with the conclusions on the safety of foam sclerotherapy as to the possible chemical lesion in distal transit/target organs.

Similarly no specific speculation on the possible foam bubbles accumulation in the lungs is possibly drawn from this study, as the mechanical passage of the SF bubbles was not quantified with this methodology; conversely the clinical/experimental study was addressed to investigate any possible residual lesion on the pulmonary capillaries/tissues after SF passage.

Though our investigations were aimed mainly at pulmonary circulation and no specific scintigraphic examination was carried out for brain/ocular circulation, the final total body scintigraphy of the second patient (step 6) did not show any residual lesion in any organs, including cerebral parenchyma.

The data from this observational study reinforce the concept of the absence of any direct chemical activity of SF within lungs and other central organs, which is coherent with the results of our scintigraphic study and with the results of other studies. ${ }^{15-19}$

Conversely all scintigraphic investigations showed a clear increase of RT accumulation in the injected areas of the lower limbs, where inflammation and sclerothrombosis obviously occurred.

\section{Conclusions}

A few conclusions may be drawn from the data collected through this scintigraphy-based preliminary study: i) definitely no damage of the sclerosant foam on the pulmonary tissues was demonstrated; ii) extemoraneous mixing of a sclerosant drug with pertechnetate ion is not a correct procedure to highlight the drug pathways in foam sclerotherapy, differently from what was shown in the past; new, alternative procedures for a stable binding between STS/POL and RT would be required if the sclerosant drug tracing within the blood stream is targeted; iii) little delayed uptake differences were shown as to the time/curves between free $\mathrm{RT}$ and SF, due to the sclerosant foam transient persistence in the peripheral vein where it was injected; iv) air-based sclerosant foam top radioactivity was reached later than $\mathrm{CO}_{2}+\mathrm{O}_{2}$ based SF, due to the early release of the RT from the bubbles in the second case; v) no significant differences were highlighted between POL and STS curves.

\section{References}

1. Wollman JC. The history of sclerosing foams. Derml Surg 2004;30:694-703.

2. Tessari L. Nouvelle technique d'obtention de la sclero-mousse. Phlébologie 2000; 53:129.

3. Tessari L, Cavezzi A, Frullini A. Preliminary experience with a new sclerosing foam in the treatment of varicose veins. Derm Surg 2001;27:58-60.

4. Rabe E, Breu F, Cavezzi A, et al. European guidelines for sclerotherapy in chronic venous disorders. Phlebology 2013;29:33854.

5. Coleridge-Smith P. Chronic venous disease treated by ultrasound guided foam sclerotherapy. EJVES 2006;32:577-83.

6. Myers KA, Jolley D, Clough A, Kirwan J. Outcome of ultrasound-guided sclerotherapy for varicose veins: medium-term results assessed by ultrasound surveillance. Eur J Vasc Endovasc Surg 2007;33:116-21.

7. Cavezzi A, Parsi K. Complications of foam sclerotherapy. Phlebology 2012;27:46-51.

8. Milleret R, Mehier H. Sclerosing foam and lung: a scintigraphic study. Abstract presented at: 21st Annual Congress of the
American College of Phlebology. Phlebology 2008;23:9.

9. Grandi L, Grandi RA, Tomasi CD, et al. Acute and chronic consequences of polidocanol foam injection in the lung in experimental animals. Phlebology 2013;28:441.

10. Saha GB. Fundamentals of nuclear pharmacy. 5th ed. New York: Springer Verlag; 2004.

11. Harden R, Alexander WD. Isotope uptake and scanning of stomach in man with 99mTc-pertechnetate. Lancet 1967;1:13057.

12. Zuckier LS, Dohan O, Li Y, et al. Kinetics of perrhenate uptake and comparative biodistribution of perrhenate, pertechnetate, and iodide by NaI symporter-expressing tissues in vivo. J Nucl Med 2004;45: 500-7.

13. Kuni CC. Manual of nuclear medicine imaging. New York: Thieme Medical Publishers; 1997.

14. Moro L, Rossi Bartoli L, Cesari M, et al. Pulmonary gas exchange after foam sclerotherapy. JAMA Dermatol 2014;150:207-9.

15. Parsi K, Exner T, Connor DE, et al. Thelytic effects of detergent sclerosants on erythrocytes, platelets, endothelial cells and microparticles are attenuated by albumin and other plasma components in vitro. Eur J Vasc Endovasc Surg 2008;36:216e23.

16. Parsi K, Exner T, Connor DE, et al. In vitro effects of detergent sclerosants on coagulation, platelets and microparticles. Eur J Vasc Endovasc Surg 2007;34,731-40.

17. Parsi K, Exner T, Low J, et al. In vitro effects of detergent sclerosants on antithrombotic mechanism. Eur J Vasc Endovasc Surg 2009;38:220-8.

18. Watkins MR. Deactivation of sodium tetradecyl sulphate injection by blood proteins. Eur J Vasc Endovasc Surg 2011; 41:521-5.

19. Tessari L, Izzo M, Cavezzi A, et al. Timing and modality of the sclerosing agents binding to the human proteins: laboratory analysis and clinical evidences. Venis and Lymphatics 2014;3:3275. 